teten b-Maxima der Sternhäufigkeit in Blei und Eisen stellen Übergangseffekt der Photonenkomponente dar.

Bei der experimentellen Durchführung der Arbeit haben Frl. E. B a r t s c h a t, die Herren G. K u h n, W. B r a u n, E. W e b e r, O. E b e r t wertvolle Hilfe geleistet. Zu besonderem Dank ist der eine von uns (E.Sch.) der Fa.
E. Leitz, Wetzlar, und Herrn Professor B e rg man n für die Überlassung eines zusätzlichen kompletten Ortholux-Mikroskopes mit Spezial-Zubehör, und für die Entwicklung besonderer Kernspur-Objektive verpflichtet. Ferner dankt er der Notgemeins chaft der Deutschen Wissenschaft für eine seit dem Jahre 1938 zur Auswertung von Kernspurplatten überlassene Mikroskop-Ausrüstung.

\title{
Solare Ultrastrahlung und Erdmagnetfeld
}

\author{
Von ARNulf Schlüter \\ Aus dem Max-Planck-Institut für Physik, Göttingen \\ (Z. Naturforschg. 6 a, 613-618 [1951]; eingegangen am 24. August 1951)
}

\section{Herrn Professor E rich Rege ner zum 70. Geburtstag}

\begin{abstract}
Durch numerische Integration von 20 Bahnen geladener Teilchen im Felde eines magnetischer Dipols wurde das Netz der von S törmer berechneten Bahnen, die von der im geomagnetischen Äquator angenommenen Sonne kommen, für den interessierenden Impulsbereich von einigen $10^{9} \mathrm{eV} / c$ soweit verfeinert, daß einige quantitative Schlüsse über die theoretische Verteilung der Ultrastrahlung auf der Erde gezogen werden können. Es ergibt sich in mittleren Breiten ein ausgeprägtes Maximum gegen $9 \mathrm{~h}$ Ortszeit (für positive Teilchen) und ein verbotener Bereich in der Nähe der Pole. Durch Näherungsbetrachtungen, die durch die Ergebnisse der numerischen Rechnung begründet werden, werden die Schlüsse auf noch niedrigere Energien und auf andere Deklination der Sonne ausgedehnt. Ein Teil cler experimentellen Befunde bei den beobachteten solaren Ultrastrahlungsausbrüchen steht in Widerspruch zu den Erwartungen nach der Störmerschen Theorie.
\end{abstract}

$\mathrm{D}$ ie zuerst von Forbush und Ehmert gemessenen und später von zahlreichen anderen Beobachtern bestätigten Anstiege der Intensität der Ultrastrahlung in See- und Berghöhe, die gleichzeitig oder kurz nach chromosphärischen Eruptionen auf der Sonne auftraten, haben sichergestellt, daß die Sonne mindestens zeitweise Ultrastrahlung erzeugt. Hierdurch hat die Frage nach den Bahnen geladener Teilchen, die von der Sonne her auf die Erde treffen, erneut an Interesse gewonnen.

Wir nehmen an, daß das Erdmagnetfeld sich durch ein Dipolfeld vom Momente $M=8,4 \cdot 10^{25} \mathrm{~cm}^{5 / 2} \mathrm{~g}^{1 / 2}$ $\mathrm{sec}^{-1}$ genügend genau annähern läßt. Dann können wir aus der Tatsache, daß bisher noch kein solcher Ultrastrahlungsanstieg am geomagnetischen Äquator beobachtet ist (nämlich in Huancayo, Peru, geomagnetische Breite $\Phi=0,6^{\circ} \mathrm{S}$ ), eine obere Grenze für den interessierenden Impulsbereich der solaren Partikel angeben. Einfach geladene Teilchen ${ }^{1}$ von einem Impuls $P>10,4 \cdot 10^{9} \mathrm{eV} / c \quad(c=$ Lichtgeschwindigkeit $)$ können den Äquator erreichen, und solche mit einem Impuls $P>15,5 \cdot 10^{9} \mathrm{eV} / c$ können dort senkrecht ein-

1 Wir werden im folgenden immer annehmen, daß die Teilchen einfach geladen sind. fallen. Man wird daher annehmen, daß die solaren Ultrastrahlungsteilchen einen Impuls von weniger als $10^{10} \mathrm{eV} / c$ besitzen. Andererseits folgt daraus, daß diese Teilchen oder ihre Folgeprodukte in Seehöhe überhaupt beobachtet werden können, daß sie sicher einen Impuls von einigen $10^{9} \mathrm{eV} / c$ besessen haben.

Nun hat S tö r m e r ${ }^{2}$ bereits im Jahre 193672 Bahnen ${ }^{3}$ von Teilchen berechnet, die im Unendlichen auf dem geomagnetischen Äquator mit verschiedenen Drehimpulsen starten. Da Bahnen verschiedenen Impulses durch eine Transformation des Längenmaßstabes ineinander übergeführt werden können, genügt es, sie für einen bestimmten Impuls zu berechnen. Zweckmäßig ist es dabei, alle Längeneinheiten in der Störmerschen Einheit $\sqrt{e M / c P} \quad(e=$ Elementarladung) zu messen, und statt der Zeit bei der Bahnberechnung die Bogenlänge in diesen Einheiten als Parameter zu benutzen. Für Teilchen großen Impulses $P$ wird die Störmersche Länge klein, der Radius der Erde in Störmerschen Einheiten gemessen $(A)$ also groß. Es gilt die Beziehung

$$
P=A^{2} \cdot 62,1 \cdot 10^{9} \mathrm{eV} / c \text {. }
$$

\footnotetext{
2 C. S t ö r m e r, Astrophysic. Norv. 2, 1 [1936].

3 Wegen der Symmetrie zum geomagnetischen Äquator entspricht dies insgesamt 132 Bahnen.
} 


\begin{tabular}{|c|c|c|c|cc|}
\hline $\begin{array}{c}A \\
\text { in Störmereinh. } 10^{9} \mathrm{eV} / c\end{array}$ & $v_{0}$ & $v_{0}{ }^{\prime}$ & $v_{1}$ & \\
\hline \multicolumn{2}{|c|}{} & & $P$ & & \\
0,05 & 0,16 & $72^{\circ}$ & $72^{\circ}$ & $78^{\circ}$ & \\
0,1 & 0,62 & $63^{\circ}$ & $63^{\circ}$ & $73^{\circ}$ & \\
0,15 & 1,4 & $56^{\circ}$ & $57^{\circ}$ & $69^{\circ}$ & \\
0,2 & 2,5 & $49^{\circ}$ & $51^{\circ}$ & $66^{\circ}$ & $\left(65^{\circ}\right)$ \\
0,3 & 5,6 & $35^{\circ}$ & $39^{\circ}$ & $63^{\circ}$ & $\left(59^{\circ}\right)$ \\
0,4 & 9,9 & $12^{\circ}$ & $27^{\circ}$ & $62^{\circ}$ & $\left(54^{\circ}\right)$ \\
0,5 & 15,5 & - & $0^{\circ}$ & $64^{\circ}$ & $\left(57^{\circ}\right)$ \\
0,6 & 22,3 & - & - & $67^{\circ}$ & $\left(53^{\circ}\right)$ \\
0,8 & 39,6 & - & - & $74^{\circ}$ & $\left(63^{\circ}\right)$ \\
1,0 & 62,1 & - & - & $90^{\circ}$ & $\left(66^{\circ}\right)$ \\
\hline
\end{tabular}

Tab. 1. $A=$ Erdradius; $P=$ Impuls pro Elementarladung; $v_{0}=$ niedrigste erlaubte geomagnetische Breite; $v_{0}{ }^{\prime}=$ niedrigste Breite, bei der Einfall aus der Meridianebene möglich ist; $v_{1}=$ höchste Breite, zu der Teilchen gelangen können, die im Unendlichen auf dem Äquator starten $\left(n=90^{\circ}\right)$. In Klammern Breite des Auftreffpunktes der polnächsten gerechneten Bahn.

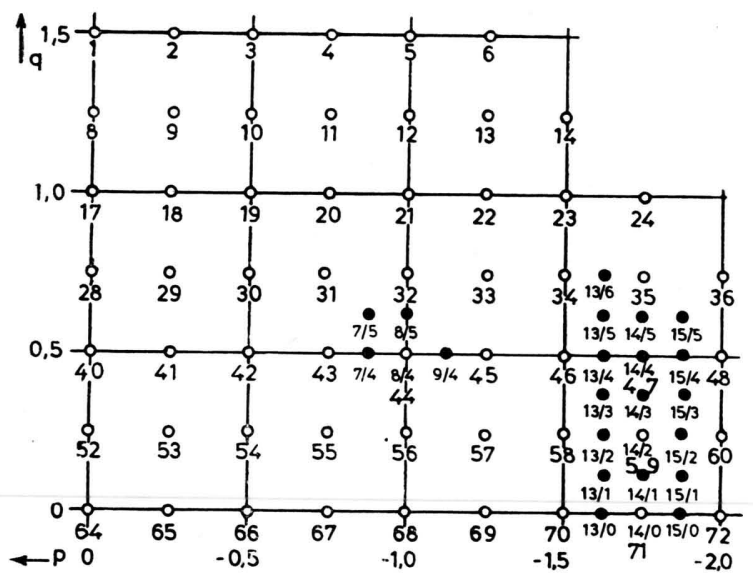

Abb. 1. Anfangsbedingungen der gerechneten Bahnen. Störmersche Bezeichnung durch laufende Nummern (Bahnen mit positivem $p$ sind weggelassen). Unsere Bezeichnung durch $-8 p$ und $8 q$ für die Bahnen im interessierenden Bereich.

Wir werden im Anschluß an Störmer $A$ als natürliches Maß für den Impuls der Teilchen verwenden.

In Tab. 1 ist der Zusammenhang zwischen $P$ und $A$ angegeben.

Von den 72 Störmerschen Bahnen gelangen nun nur 2 bis auf eine Entfernung von $A=0,4$ an den Dipol heran, d. h. bei $P=9,9 \cdot 10^{9} \mathrm{eV} / c$ noch auf die Erde. Es wurde durch numerische Integration von 20 weiteren Bahnen das Netz von Bahnen verdichtet für den Bereich von Anfangsbedingungen, in dem Bahneu mit $A \leq 0,4$ nach graphischer Interpolation zwischen den Störmerchen Bahnen möglich erscheinen. Abb. 1 zeigt die Lage der Anfangsbedingungen für die mei-

\begin{tabular}{|c|c|c|c|c|}
\hline \multicolumn{2}{|c|}{ Bezeichnung } & \multicolumn{2}{|c|}{ Anfangsbedingung } & \multirow[b]{2}{*}{$A_{\min }$} \\
\hline Störmer & wir & $-p$ & $q$ & \\
\hline & $\begin{array}{l}7 / 4 \\
7 / 5\end{array}$ & 0,875 & $\begin{array}{l}0,500 \\
0,625\end{array}$ & $\begin{array}{l}0,280 \\
0,197\end{array}$ \\
\hline $44=-92$ & $\begin{array}{l}8 / 4 \\
8 / 5\end{array}$ & 1,000 & $\begin{array}{l}0,500 \\
0,625\end{array}$ & $\begin{array}{l}0,250 \\
0,275\end{array}$ \\
\hline & $9 / 4$ & 1,125 & 0,500 & 0,355 \\
\hline & $\begin{array}{l}13 / 0 \\
13 / 1 \\
13 / 2 \\
13 / 3 \\
13 / 4 \\
13 / 5 \\
13 / 6\end{array}$ & 1,625 & $\begin{array}{l}0 \\
0,125 \\
0,250 \\
0,375 \\
0,500 \\
0,625 \\
0,750\end{array}$ & $\begin{array}{l}0,476 \\
0,477 \\
0,479 \\
0,488 \\
0,489 \\
0,315 \\
0,427\end{array}$ \\
\hline $47=-95^{*}$ & $\begin{array}{l}14 / 1 \\
14 / 3 \\
14 / 4 \\
14 / 5\end{array}$ & 1,750 & $\begin{array}{l}0,125 \\
0,375 \\
0,500 \\
0,625\end{array}$ & $\begin{array}{l}0,455 \\
0,363 \\
0,174 \\
0,431\end{array}$ \\
\hline$* *$ & $\begin{array}{l}15 / 0 \\
15 / 1 \\
15 / 2 \\
15 / 3 \\
15 / 4 \\
15 / 5\end{array}$ & 1,875 & $\begin{array}{l}0 \\
0,125 \\
0,250 \\
0,375 \\
0,500 \\
0,625\end{array}$ & $\begin{array}{l}0,433 \\
0,431 \\
0,468 \\
0,407 \\
0,222 \\
1,190\end{array}$ \\
\hline
\end{tabular}

* Die Berechnung dieser besonders langen Bahn wurde von Störmer vor Erreichen des Umkehrpunktes abgebrochen. Die Bahn wurde von uns vollständig berechnet.

** Diese Bahn erreicht zunächst in südlicher Breite ein Minimum von $A=0,419$, entfernt sich dann bis $A=$ 0,67 , um in nördl. Breite umzukehren.

Tab. 2.

sten von Störmer gerechneten und für unsere Bahnen. $p$ und $q$ sind rechtwinklige Koordinate, die die Durchstoßpunkte der Bahnen durch eine Ebene senkrecht zur Flugrichtung außerhalb des Erdmagnetfeldes angeben. $q$ ist die Komponente parallel zur Dipolachse, so daß der Südpol des Dipols oben liegt, $p$ ist senkrecht dazu und wächst von Ost nach West. Alle Teilchen starten parallel zueinander; die Bahn mit $p=$ $q=0$ zielt genau auf den Dipol. Es ist zu beachten, daß alle Bilder und Angaben im Anschluß an Störmer für negative Teilchen gelten. Die hohlen Kreise geben die Anfangswerte und die Bahnnummern der Störmerschen Bahnen, die gefüllten Kreise unsere Bahnen, die wir in der Form $-8 p / 8 q$ bezeichnet haben. Die numerischen Integrationen wurden genau in der von Störmer ${ }^{2}$ angegebenen Weise durchgeführt.

Tab. 2 gibt die Minimaldistanzen der von uns gerechneten und der interessierenden Störmerschen Bahnen an. Bei letzteren ist außer der Nummer der Bahn 
In Tab. 3 geben wir einen Auszug, der die ersten Nullbahnen einer Familie solcher Bahnen enthält.

Die Bahn 1 hat den glattesten Verlauf, die folgenden Bahnen durchschneiden die Äquatorialebene je einmal mehr. Das erste Bündel in der Gegend der Bahn 7/5 gehört offenbar zu der ersten Nullbahn, während das zweite Bündel $(13 /-5,14 /-3,14 /-4)$ wohl zu der zweiten Nullbahn gehört.

Die Tatsache, daß in dem interessierenden Impulsbereich die meisten Bahnen in der Nähe einer Nullbahn liegen, erlaubt, einige weitere Schlüsse zu ziehen. Wir gehen von der (bereits einmal integrierten) $\varphi$-Komponente der Bewegungsgleichung aus:

$$
\frac{\mathrm{d} \varphi}{\mathrm{d} s}=\frac{1}{r^{3}}+\frac{p \sin n}{r^{2} \cos ^{2} v}
$$

(Störmersche Längeneinheiten, $\varphi=$ geomagnetische Länge, $s=$ Bogenlänge auf der Bahn, $r=$ räumlicher Abstand vom Dipol, $p \cdot \sin n=2 \gamma=$ Drehimpuls um die Dipolachse im Unendlichen, $n=$ Poldistanz $^{4} \mathrm{im}$ Unendlichen, $v=$ geomagnetische Breite im jeweiligen Punkte der Bahn).

Die Änderung der geomagnetischen Länge des Punktes längs der Bahn hängt mit dem Winkel $\Theta$ zwischen Meridianebene und Bahntangente zusammen:

$$
\sin \Theta=r \cos v \frac{\mathrm{d} \varphi}{\mathrm{d} s} .
$$

Es ist nun $2 \gamma=p \cdot \sin n$ stets beschränkt, so daß $\gamma \geq-1$; aus $|\sin \Theta| \leq 1$ folgt daher aus (1) die Größe des für Teilchen aus beliebiger Richtung verbotenen Bereiches, wenn man $r$ gleich dem dem jeweiligen Impulse entsprechenden $A$ setzt. So folgt die Größe des in Tab. 1 angegebenen und in Abb. 2-4 und $6-8$ eingezeichneten verbotenen Bereiches um den Äquator herum. Damit das Teilchen aus der Meridianebene auf der Erde einfallen kann - eine notwendige Bedingung für Einfall aus dem Zenit muß im Auftreffpunkt $\sin \Theta=\mathrm{d} \psi / \mathrm{d} s=0$ gelten. Dies gibt für den Extremfall $\gamma=-1$ die höhere in Tab. 1 ebenfalls angegebene Mindestbreite $v_{0}{ }^{\prime}$. Für die besonders interessierenden Teilchen, die zu einem der Bündel in der Nähe einer Nullbahn gehören, liegt $p$ bzw. $\gamma$ nun in einem ganz engen Bereich. In der Abb. 6 sind als gestrichelte Kurven die nördliche und südliche Grenze des Bereiches angegeben, in dem Teilchen mit dem Drehimpuls der beiden ersten Nullbahnen überhaupt gelangen können - innerhalb dieser Bereiche fallen wegen der nicht berücksichtig-

4 Der bisher betrachtete Fall einer im Äquator stehenden Quelle entspricht $n=90^{\circ}$. ten Schattenwirkung der Erde noch weitere Bahnen aus. Ausgezogen sind die Kurven, auf die Teilchen des Drehimpulses der drei ersten Nullbahnen vertikal einfallen können. Die entsprechenden Kurven für die weiteren Nullbahnen liegen noch näher an der $\mathrm{Ab}$ schneidegrenze. Das Bild ist wegen der Symmetrie des Problems $\left(n=90^{\circ}\right.$ !) nur für nördliche Breiten gezeichnet und symmetrisch zum Äquator ergänzt zu denken. Es fällt besonders auf, daß auch eine höchste Breite für jeden Impuls existiert, polwärts von denjenigen Teilchen, die mit $n=90^{\circ}$ im Unendlichen starten, die Erde nicht erreichen können. Solch eine nördliche Grenze existiert allgemein für $A<1$ und folgt wieder aus den Gl. (1) und (2), wenn man durch

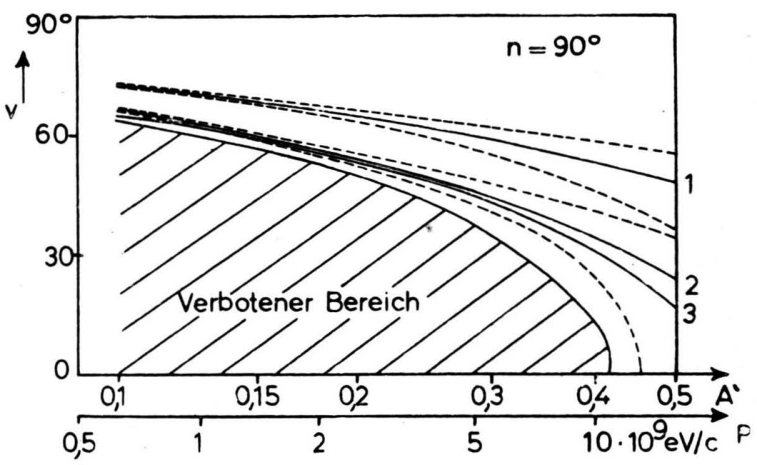

Abb. 6. Geomagnetische Breite des Auftreffpunktes der ersten Nullbahnen in Abhängigkeit vom Impuls für die Deklination $0^{\circ}$ der Quelle.

graphische Interpolation aus den gerechneten Bahnen das absolut kleinste $p$ bzw. $\gamma$. bestimmt, das ein bestimmtes $A$ noch erlaubt. Die polwärtige Abschneidegrenze ist als $v_{1}$ ebenfalls in Tab. 1 angegeben. Als Anhaltspunkt, wie weit diese obere Grenze wirklich erreicht wird, ist zugleich in Klammern die geomagnetische Breite der polnächsten gerechneten Bahnen angegeben. Eine geomagnetische Breite von $80^{\circ}$ kann z. B. von Teilchen, die aus der im Äquator stehenden Sonne kommen, nur erreicht werden, wenn sie einen Impuls von $P \leq 10^{8} \mathrm{eV} / c$ oder $P>5 \cdot 10^{10}$ $\mathrm{eV} / c$ besitzen. Eine solche Breite ist also für den gesamten uns interessierenden Impulsbereich verboten.

Da $S$ törme ${ }^{5}$ die Anfangswerte für einige Nullbahnen auch für $n \neq 90^{\circ}$ berechnet hat, kann auch über den Fall, daß die Sonne außerhalb des Äquators steht, etwas gesagt werden. Für $n=80^{\circ}$ (nördliche geomagnetische Deklination $10^{\circ}$ ) ist in der Abb. 7 für die Nullbahnen, die aus den beiden ersten für $n=$

5 C. S törm e r, Astrophysica Norv. 1, 115 [1935]. 


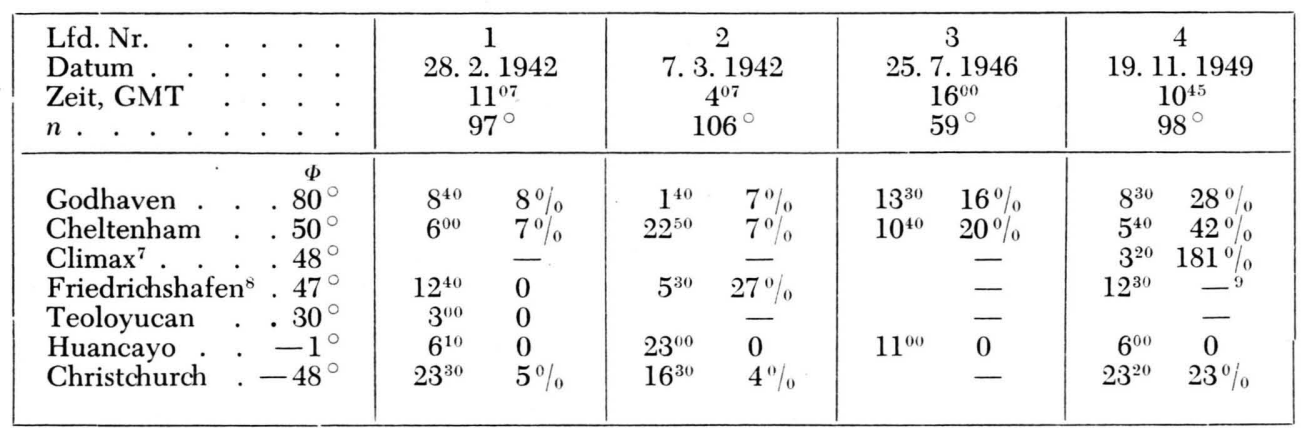

Tab. 4. Oben: Datum und Weltzeit des Beginns der 4 Ereignisse sowie geomagnetische Poldistanz der Sonne $(n)$; links: Beobachtungsort mit geomagnetischer Breite $(\Phi)$; Inhalt: mittlere geomagnetische Ortszeit und prozentualer Anstieg der Intensität in einer mit $10 \mathrm{~cm} \mathrm{~Pb}$ gepanzerten Ionisationskammer. 0: kein Anstieg; —: keine Messung.

$90^{\circ}$ betrachteten stetig hervorgehen, die Auftreffbreite in Abhängigkeit vom Impuls aufgetragen. Das Bild sieht der Abb. 6 noch recht ähnlich; die beiden Bündel 1 sind nach Norden und die Bündel 2 nach Süden verschoben. Das heißt, daß der Hauptteil der von der Sonne kommenden Ultrastrahlung im Sommer in etwas höhere Breite gelangt als im Winter. Für große Deklinationen der Sonne ist der Schluß allerdings nicht ganz sicher, da sich die Besetzungszahl der einzelnen Bündel ändern können. Für einen festen Impuls (entsprechend $A=0,3$ ) ist in der Abb. 8 die geomagnetische Breite des Durchstoßpunktes der Nullbahnen durch die Nordhalbkugel der Erde in Abhängigkeit von der Poldistanz $n$ der unendlich entfernten Quelle aufgetragen, die in der für $A=0,3$ recht guten Näherung berechnet wurden, daß $\Theta=0$ gesetzt wurde. Die drei numerierten Durchgänge durch $n=90^{\circ}$ entsprechen den ebenso numerierten Kurven der Abb. 6. Man sieht, daß für eine Poldistanz von $n=65^{\circ}$ (Nordsommer) nur eine einzige Nullbahn existiert, die auf der Nordhalbkugel auftrifft, während die Südhalbkugel (und entspr. die Nordhalbkugel im Nordwinter) nicht erreicht wird. Trotz der Unsicherheit über die Besetzung der zu den Nullbahnen gehörigen Bündel (die nur durch numerische Integration weiterer Bahnen beseitigt werden können), wird man vermuten, daß für den interessierenden Impulsbereich im Nordsommer in mittleren nördlichen Breiten ein recht scharfer tageszeitlicher Effekt vorhanden ist, während alle südlichen Breiten verboten sind - und entsprechend umgekehrt im Nordwinter.

$73000 \mathrm{~m}$ über Seehöhe; zusätzliche Schirmung durch $12,6 \mathrm{~cm} \mathrm{Fe}$.

8 Koinzidenzanlage.

9 Anstieg von mehreren anderen deutschen Stationen und in Manchester registriert.

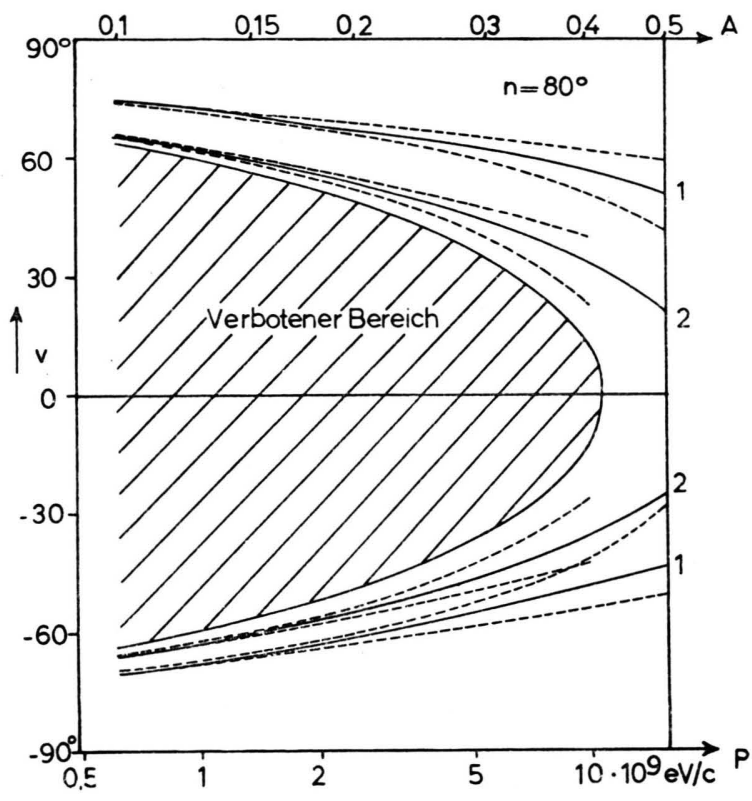

Abb. 7. Wie Abb. 6 für Deklination $10^{\circ} \mathrm{N}$ der Quelle.

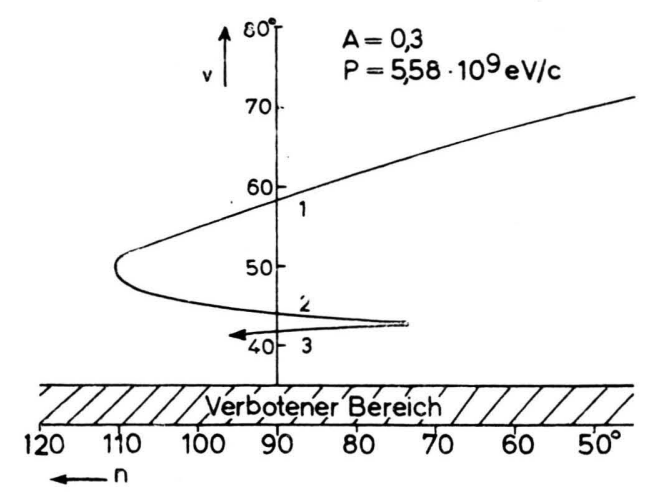

Abb. 8. Geomagnetische Breite des Auftreffpunktes der ersten Nullbahnen in Abhängigkeit von der Poldistanz $n$ der Quelle bei festem Impuls. Der Nullpunkt der $v$-Skala ist unterdrückt. 
Der Vergleich mit der Erfahrung soll hier nicht im einzelnen diskutiert werden. Es soll jedoch auf einige Schwierigkeiten der theoretischen Deutung der Beobachtungen der solaren Ultrastrahlungsausbrüche hingewiesen werden. In Tab. 4 ist eine Übersicht über die vier großen bisher beobachteten Effekte ${ }^{6}$ gegeben.

Überraschend ist vor allem, daß in Godhavn (Grönland) alle Effekte, sogar der zweite (negative Deklination der Sonne!) gemessen werden konnten. Auch

$6 \mathrm{~S}$. E. Forbush, T. B. Stinchcomb u. M. S chein, Physic. Rev. 79, 501 [1950]. A. Ehmert, Z. Naturforschg. 3a, 264 [1948]. andere Registrierungen (z. B. Nr. 4 in Deutschland kurz nach Mittag und die siebenstündige Dauer dieses Effektes in Climax) sind schlecht zu verstehen. Man wird schließen müssen, daß die Voraussetzung der Rechnung, daß allein ein Dipolfeld der Erde für die Ablenkung verantwortlich ist, nicht genügend genau erfüllt ist.

Verfasser dankt Herrn Professor B i e r m a n n für seine Unterstützung und sein großes Interesse, Herrn $\mathrm{Hell}$ wig für manche Hilfe und insbesondere Fräulein Kreut ziger und Herrn K a t e r b a ch für die Durchführung des größten Teils der langwierigen numerischen Rechnungen.

\title{
Ein ortszeitlicher weltweiter Tagesgang der kosmischen Ultrastrahlung bei geringen erdmagnetischen Störungen durch Korpuskeln ${ }^{1}$
}

\author{
Von A. Ehmert und A. Sittkus \\ Aus der Forschungsstelle für Physik der Stratosphäre in der Max-Planck-Gesellschaft, Weißenau, \\ und dem Physikalischen Institut der Universität Freiburg i. Br. \\ (Z. Naturforschg. 6 a, 618-622 [1951]; cingegangen am 16. April 1951)

\section{Erich Regener zum 70. Geburtstag}

\begin{abstract}
Bei schwachen erdmagnetischen korpuskelbedingten Störungen tritt in der Intensität der kosmischen Ultrastrahlung ein charakteristischer Tagesgang auf. Der Vergleich der gleichzeitigen Auswirkung in Ionisationskammern und Zählrohrkoinzidenzgeräten zeigt, daß wahrscheinlich zwei Vorgänge zusammenspielen. Das Auftreten und sogar in hohem Maß der Verlauf dieser Tagesgänge sind weltweite Erscheinungen. Aber der Verlauf ist an die Ortszeit gebunden.
\end{abstract}

$\mathrm{D}$ ie Verfeinerung der Registriermethoden erlaubt nunmehr schon kleine Schwankungen der Intensität und der Teilchenzahl der kosmischen Ultrastrahlung sofort zu erkennen, wenn dieselben in der Größenordnung von $1 \%$ sind. S it t k u s ${ }^{2}$ verwendet eine 500-Liter-Ionisationskammer mit $10 \mathrm{~cm}$ dickem Eisenpanzer, die auf dem Turm der Freiburger Universität aufgestellt ist, und registriert größere Hoffmannsche Stöße in dieser Kammer gesondert, so daß ihre Extraionisationen für jeden Stundenwert von der Gesamtionisation abgezogen werden können und nicht als pseudostatistische Schwankungen in Erscheinung treten. Die Ionisationswerte sind deshalb ein gutes $\mathrm{Maß}$ für die Zahl der Einzelteilchen mit einer statistischen

${ }^{1}$ Gekürzte Mitteilung: Physik. Verhandlungen, Heft 2, 29 [1951].

2 A. S it tk u s, Z. Naturforschg. 1, 204 [1946]. Die Ergebnisse werden seit Januar 1950 laufend im Sonnenzirkular des Fraunhofer-Institutes in Freiburg i. Br. veröffentlicht.
Streuung des Stundenmittels von etwa 1\%o. Ehmert benutzt eine Zählrohranlage und registriert getrennt die Zweifachkoinzidenzen durch zwei Zählflächen von je $750 \mathrm{~cm}^{2}$. Im Mittel beider Anlagen macht der mittlere statistische Fehler eines Stundenmittels $6 \%$ der Koinzidenzzahl aus.

In beiden Registrierungen im Jahr 1950 fiel eine Anzahl von Tagen durch einen die statistische Schwankung mehrfach übersteigenden Tagesgang mit einem mittäglichen Maximum auf. Die deutlichsten Beispiele zeigen die Abb. 1 bis 3. Dort ist der Verlauf der Koinzidenzzahlschwankungen in Weißenau (W) und der Ionisationsschwankungen in Freiburg (Fr) nach Korrektur des Einflusses von Luftdruckschwankungen aufgetragen. Schwankungen der Außentemperatur scheiden als Ursache dieser Tagesgänge aus, wie ein Vergleich mit den bei gleichen meteorologischen Verhältnissen ungestörten Tagen einerseits und mit den statistischen Ergebnissen über Untersuchungen des 